\title{
Semi-dynamic Algorithms for Strongly Chordal Graphs
}

\author{
Md. Zamilur Rahman and Asish Mukhopadhyay \\ School of Computer Science \\ University of Windsor
}

February 19, 2020

\begin{abstract}
There is an extensive literature on dynamic algorithms for a large number of graph theoretic problems, particularly for all varieties of shortest path problems. Germane to this paper are a number fully dynamic algorithms that are known for chordal graphs. However, to the best of our knowledge no study has been done for the problem of dynamic algorithms for strongly chordal graphs. To address this gap, in this paper, we propose a semi-dynamic algorithm for edgedeletions and a semi-dynamic algorithm for edge-insertions in a strongly chordal graph, $G=$ $(V, E)$, on $n$ vertices and $m$ edges. The query complexity of an edge-deletion is $O\left(d_{u}^{2} d_{v}^{2}(n+m)\right)$, where $d_{u}$ and $d_{v}$ are the degrees of the vertices $u$ and $v$ of the candidate edge $\{u, v\}$, while the query-complexity of an edge-insertion is $O\left(n^{2}\right)$.
\end{abstract}

\section{Introduction}

Let $G=(V, E)$ be a connected graph on $n(=|V|)$ vertices with $m(=|E|)$ edges. $G$ is chordal if it has no induced chordless cycle of size greater than three. An alternate characterization that is more apposite for this paper is there exists a perfect elimination ordering of its vertices. We will represent a chordal graph by a clique tree (see [1]).

Strongly chordal graphs which are a subclass of chordal graphs was introduced into the graph theory literature by Farber in [3. $G$ is strongly chordal if there exists a strong elimination ordering of its vertices, a generalization of the notion of perfect elimination ordering for chordal graphs.

While the problems of finding a minimum weight dominating set and an independent dominating set in vertex-weighted chordal graphs are NP-hard, Farber again 5 s showed that these can be solved in linear time for strongly chordal graphs, provided a strong elimination ordering of the vertices of the graph is known.

There is an extensive literature on dynamic algorithms for a large number of graph theoretic problems, particularly for all varieties of shortest path problems. Germane to this paper are a number fully dynamic algorithms that are known for chordal graphs [6, 8]. However, to the best of our knowledge no study has been done for the problem of dynamic algorithms for strongly chordal graphs.

To address this gap, in this paper, we propose a semi-dynamic algorithm for edge-deletions and a semi-dynamic algorithm for edge-insertions in a strongly chordal graph.

The rest of the paper is structured as follows. In the next section, we explain the design of semi-dynamic algorithm for deletions. In the following section 3, we discuss the complexity of 
the proposed semi-dynamic algorithms. Finally, section 4 contains concluding remarks and open problems.

\section{Semi-dynamic Algorithm for Deletions}

Let $G=(V, E)$ be a strongly chordal graph and $C$ an even cycle of size six or greater in $G$. A chord $\{u, v\}$ of $C$ is a strong chord if a shortest distance between $u$ and $v$ along $C, d_{C}(u, v)$, is odd. The deletion algorithm is based on the following characterization of a strongly chordal graph.

Theorem 1 [4 A graph $G$ is strongly chordal if and only if it is chordal and every even cycle of length at least 6 in $G$ has a strong chord.

Let $e=\{u, v\}$ be an arbitrary edge of $G$. Then $e$ can be deleted from $G$ provided $G-e$ remains chordal and it is not the only strong chord of a six-cycle. The check for chordality exploits the following theorem.

Theorem 2 [6] Let e be an edge of a chordal graph $G$. Then $G-e$ remains chordal if and only if $G$ has exactly one maximal clique containing e.

Since strongly chordal graphs are a subclass of chordal graphs, a clique tree data structure, $T$, representing $G$ is used to check for the chordality condition.

Consider the chordal graph shown in Figure 1(a) that has three maximal cliques $v_{1} v_{2} v_{3}, v_{1} v_{3} v_{4} v_{5}$, and $v_{1} v_{5} v_{6}$. Each maximal clique is represented by a node in the clique tree $T$ and the weight of each edge is the size of the overlap of the two maximal cliques that it joins. To obtain a clique tree $T$ from $G$, we use an expanded version of the Maximum Cardinality Search (MCS) algorithm by Blair and Peyton [2. If an edge $e$ is present in two or more clique tree nodes, then we cannot

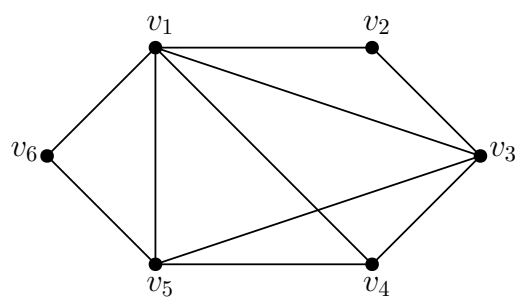

(a) A chordal graph $(G)$

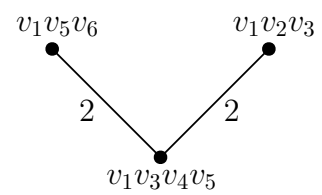

(b) A clique tree $(T)$

Figure 1: An example

delete $e$ as its deletion will violate the chordality property of $G$. For instance, we cannot delete the edge $\left\{v_{1}, v_{5}\right\}$ or the edge $\left\{v_{1}, v_{3}\right\}$ from $G$ (see Figure 3) because with the deletion of either of these two edges $G$ will cease to be chordal. Thus by maintaining the clique tree data structure $T$, we can determine if an edge can be deleted without violating chordality. This is done as follows. For each node in $T$, we compute the intersection of the node (a maximal clique contains two or more vertices of $G$ ) with the edge $e$. If we find $T$ has exactly one node containing the end-points of $e$, then we continue and check if the deletion preserves strong chordality.

As explained earlier, an edge $e$ can be deleted if and only it is not the only strong chord of a six-cycle. For instance, consider the strongly chordal graph shown in Figure 2 , The edge $\left\{v_{0}, v_{5}\right\}$ 
(shown as a dashed line segment) splits the 8-cycle, $\left\langle v_{0}, v_{1}, v_{2}, v_{3}, v_{4}, v_{5}, v_{6}, v_{7}, v_{0}\right\rangle$, into a 4-cycle, $\left\langle v_{0}, v_{5}, v_{6}, v_{7}, v_{0}\right\rangle$, and a 6-cycle, $\left\langle v_{0}, v_{1}, v_{2}, v_{3}, v_{4}, v_{5}, v_{0}\right\rangle$. The addition of a strong chord $\left\{v_{1}, v_{4}\right\}$ (shown as a dashed line segment) splits the 6-cycle, $\left\langle v_{0}, v_{5}, v_{4}, v_{3}, v_{2}, v_{1}, v_{0}\right\rangle$, into two 4-cycles, $\left\langle v_{0}, v_{1}, v_{4}, v_{5}, v_{0}\right\rangle$ and $\left\langle v_{1}, v_{2}, v_{3}, v_{4}, v_{1}\right\rangle$. Alternately, we could interpret $\left\{v_{0}, v_{5}\right\}$ as a strong chord of the 6-cycle $\left\langle v_{0}, v_{7}, v_{6}, v_{5}, v_{4}, v_{1}, v_{0}\right\rangle$, post the introduction of $\left\{v_{1}, v_{4}\right\}$ as a strong chord of the initial 8-cycle.

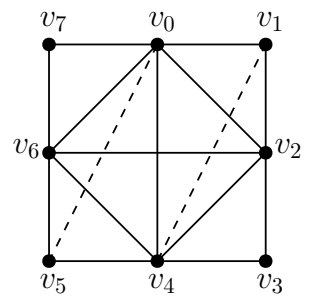

Figure 2: A strongly chordal graph

Since the addition of a strong chord splits an even cycle of size 6 or greater into two odd-length paths, it is sufficient to check for the presence of a strong chord in every even cycle of length 6 . We give a formal proof of this in an Appendix.

A potential 6-cycle of which $e=\{u, v\}$ is a strong chord is formed by disjoint pairs of chordless $P_{4}$ paths (each spanning four vertices) that go from $u$ to $v$. Thus we determine all such $P_{4}$ paths and for every disjoint pair of these we check whether $\{u, v\}$ is the only strong chord or not. If there is a strong chord other than $\{u, v\}$ in every disjoint pair of $P_{4}$ paths, then the edge $\{u, v\}$ can be deleted. On the other hand, if $\{u, v\}$ is the only strong chord for any disjoint pair of $P_{4}$ paths, then the edge $\{u, v\}$ cannot be deleted.

Now, instead of searching for $P_{4}$ paths in the entire graph, we do a local search in an induced graph, called AuxGraph. This graph is induced by a set of vertices AuxNodes that includes the closed neighbors of both the end-points of the edge $\{u, v\}$ to be deleted. Formally, we define AuxNodes $=N[u] \cup N[v]$.

Setting $u$ as the source vertex, we now perform breadth-first search in $G[$ AuxNodes] to find all $P_{4}$ paths from $u$ to $v$.

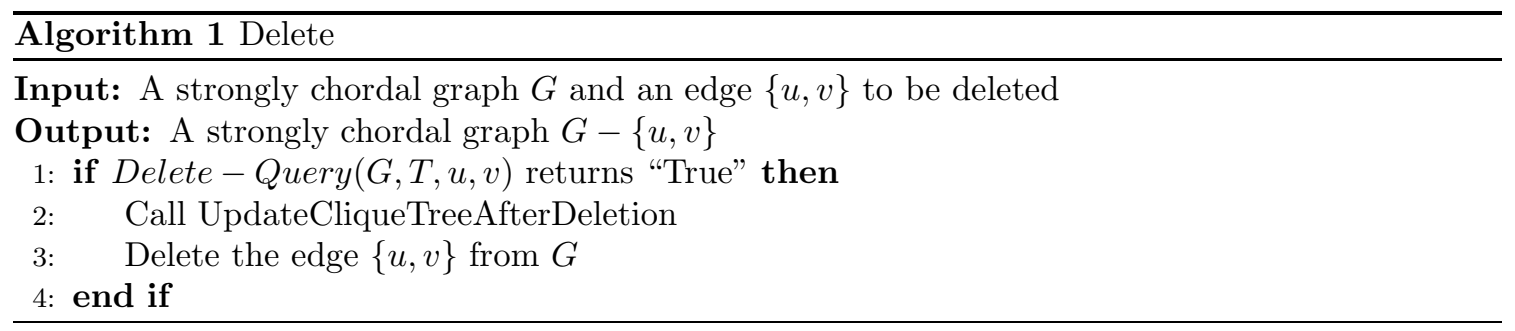

Algorithm 2 returns "True", if $\{u, v\}$ can be deleted from $G$. When the algorithm returns "True", we perform the delete operation (see Algorithm 1). After performing the delete operation, we update both the clique tree and the graph. The clique tree node that contains the edge $\{u, v\}$ can be replaced with 0,1 , or 2 nodes. The algorithm UpdateCliqueTreeAfterDeletion due to Ibarra 6] deletes the edge $\{u, v\}$ and updates $T$. 


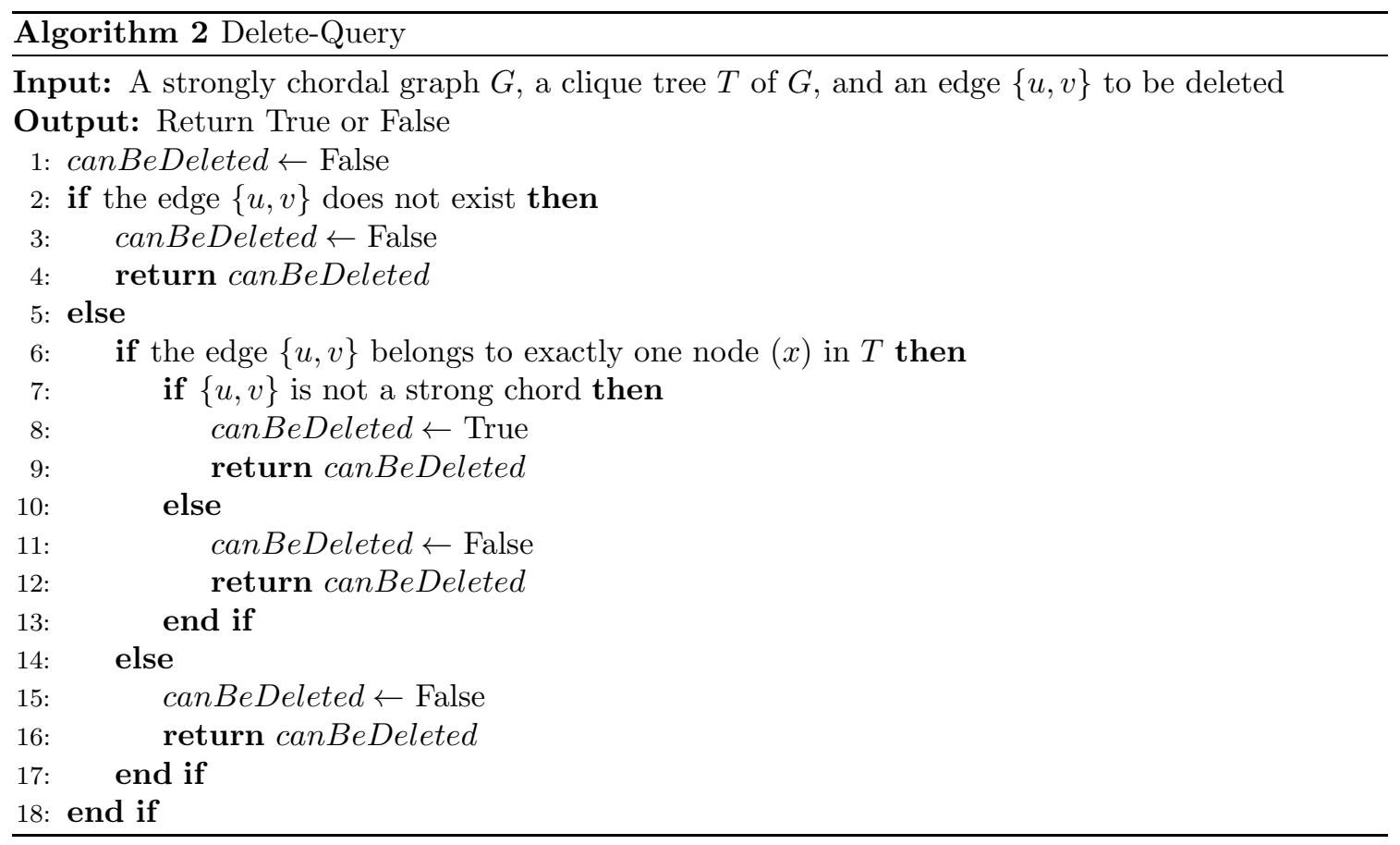

Consider the strongly chordal graph $G$ shown in Figure 1(a). Can we delete the edge $\left\{v_{1}, v_{4}\right\}$ ? At first, we check the chordality property. From the clique tree $T$ (see Figure $1(\mathrm{~b})$, we observe there is a single node containing the edge $\left\{v_{1}, v_{4}\right\}$ only. This satisfies the chordality condition and now we check the strong chordality condition. To do that, we compute the closed neighborhood of $v_{1}$ and $v_{4}$ and then compute the AuxNodes set, where AuxNodes $=N\left[v_{1}\right] \cup N\left[v_{4}\right]=\left\{v_{1}, v_{2}, v_{3}, v_{4}, v_{5}, v_{6}\right\} \cup$ $\left\{v_{4}, v_{1}, v_{3}, v_{5}\right\}=\left\{v_{1}, v_{4}, v_{2}, v_{3}, v_{5}, v_{6}\right\}$. There are two $P_{4}$ paths $\left(v_{1}-v_{2}-v_{3}-v_{4}\right.$ and $\left.v_{1}-v_{6}-v_{5}-v_{4}\right)$ between $v_{1}$ and $v_{4}$ in the AuxGraph created from the AuxNodes set. We notice that $\left\{v_{1}, v_{4}\right\}$ is the only strong chord in the AuxGraph. Hence the deletion of $\left\{v_{1}, v_{4}\right\}$ is not allowed. But in the presence of any of the other two strong chords $\left(\left\{v_{2}, v_{5}\right\}\right.$ or $\left.\left\{v_{3}, v_{6}\right\}\right)$ in the AuxGraph we will be able to delete $\left\{v_{1}, v_{4}\right\}$.

Consider another example graph, shown in Figure 3(a) where the chordality is preserved if we delete $\left\{v_{7}, v_{2}\right\}$, but the edge $\left\{v_{7}, v_{2}\right\}$ is the only strong chord for the 6-cycle $\left(v_{7}-v_{1}-v_{6}-v_{2}-\right.$ $\left.v_{5}-v_{4}-v_{7}\right)$. So, the edge $\left\{v_{7}, v_{2}\right\}$ cannot be deleted. But the edge $\left\{v_{5}, v_{4}\right\}$ can be deleted from the graph. After checking the clique tree, we see that only a single node contains the edge $\left\{v_{5}, v_{4}\right\}$. So we can proceed to check for the strong chordality condition. We observe that $\left\{v_{5}, v_{4}\right\}$ is not a strong chord and thus we can delete the edge $\left\{v_{5}, v_{4}\right\}$ and update both $T$ and $G$ (as shown in Figure 3(c) and 3(d), respectively.)

\subsection{Complexity of Deletions}

For the semi-dynamic deletion, we create a clique tree $T$ in $O(|V|+|E|)$ time, using the expanded version of the MCS algorithm. To check if the edge $\{u, v\}$ belongs to exactly one maximal clique in $T$, we perform the intersection operation between a maximal clique and the edge $\{u, v\}$. This 


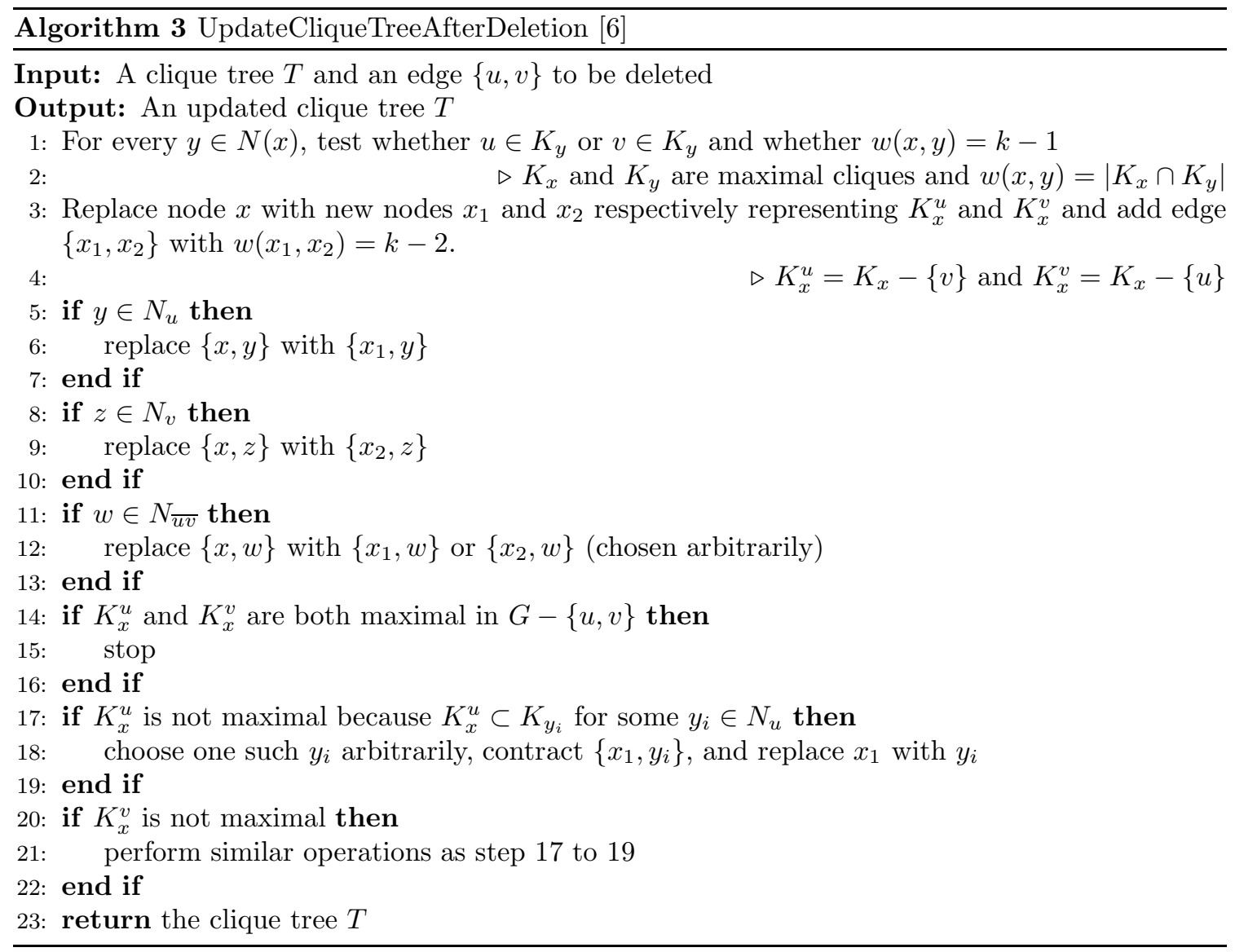

operation takes linear time. To bound the query complexity of deleting an edge $\{u, v\}$ from the strongly chordal graph, we note that this is dominated by the case of finding multiple $P_{4}$ paths between $u$ and $v$ and we have to consider these in pairs and run the breadth-first search. An upper bound on the number of pairs of $P_{4}$ paths between $u$ and $v$ is $O\left(d_{u}^{2} d_{v}^{2}\right)$, where $d_{u}$ and $d_{v}$ are the degrees of $u$ and $v$ respectively. For consider such a path from $u$ to $v$ (Fig. (4): $x$ is one of the at most $d_{u}$ vertices adjacent to $u$ and $y$ is one of the at most $d_{v}$ vertices adjacent to $v$, so that we have at most $O\left(d_{u} d_{v}\right) P_{4}$ paths from $u$ to $v$ and thus $O\left(d_{u}^{2} d_{v}^{2}\right)$ disjoint pairs of $P_{4}$ paths from $u$ to $v$.

If $|E|$ is the number of edges currently, in the strongly chordal graph, the complexity of running a breadth-first-search is $O(n+|E|)$. Since $m$ is the number of edges in the final strongly chordal graph, an upper bound on the query complexity is $O\left(d_{u}^{2} d_{v}^{2}(n+m)\right)$.

The deletion of an edge take constant time since we maintain an adjacency matrix data structure to represent $G$. This is in addition to the clique tree that we maintain. It is however possible to dispense with the adjacency matrix. In that case, there is additional work required to construct the graph $G$ from the clique tree. This can be done using a certain Running Intersection Property of chordal graphs (see [2] for details). 


\section{Semi-dynamic Algorithm for Insertions}

Let $\alpha=\left\langle v_{1}, v_{2}, \ldots, v_{n}\right\rangle$ be a strong elimination ordering of the vertices $V$ of a strongly chordal graph $G$. The neighborhood matrix $M(G)$ of $G$, based on $\alpha$, is an $n \times n$ matrix whose $(i, j)$-th entry is 1 if $v_{i} \in N\left[v_{j}\right]$ and is 0 otherwise. Let $\Delta$ be the submatrix:

$$
\Delta=\left[\begin{array}{ll}
1 & 1 \\
1 & 0
\end{array}\right]
$$

Our dynamic insertion algorithm is based on the following observation.

Observation 1 [4] The row (and column) labels of $M(G)$ correspond to a strong elimination ordering if and only if the matrix $M$ does not contain $\Delta$ as a submtraix.

The absence of $\Delta$ in $M$ implies that $M$ is totally balanced and the theorem below allows us to claim that $G$ is strongly chordal.

Theorem 3 [4] A graph $G$ is strongly chordal if and only if $M(G)$ is totally balanced.

Thus if $G$ is strongly chordal, then $G+\{u, v\}$ remains so if inserting the edge $\{u, v\}$ into $G$ does not create any $\Delta$ submatrix in $M(G+\{u, v\})$.

To insert an edge $\{u, v\}$ into $G$, we first check if it is already present in $G$. If not, we insert it if no submatrix $\Delta$ is created.

The initialization process consists of computing a strong elimination ordering $\alpha$ of an input strongly chordal graph $G$, using a recognition algorithm for strongly chordal graphs due to Farber 4 .

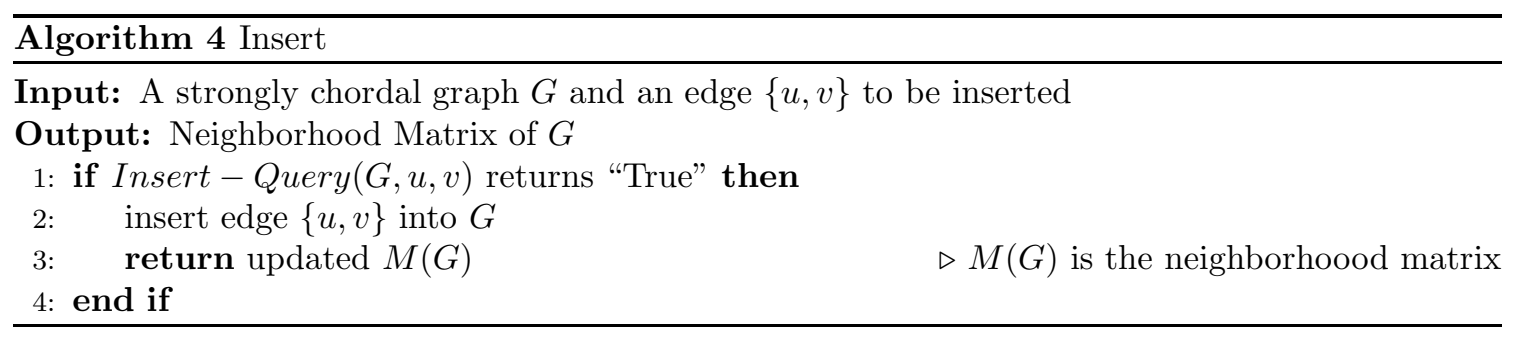

After creating a strong elimination ordering $\alpha$, we create the neighborhood matrix $M(G)$ of $G$. We also find the order of $u$ and $v$ in the ordering $\alpha$. Now we check if the insertion of an edge $\{u, v\}$ creates any $\Delta$ submatrix or not. The searching strategy is explained with the Figure 5 . Assume there is a 0 in the $i^{t h}$ row and $j^{t h}$ column. Now we want to change the entry from 0 to 1 (which corresponds to the insertion of an edge in the graph). Before changing the entry from 0 to 1 , we need to check if that creates any $\Delta$ submatrix in the $M(G)$ or not. To check the presence of $\Delta$ submatrix in $M(G)$, we need to check in three different directions from the $(i, j)$-th position. We need to check in the upward direction from $(i, j)$ to $(1, n)$, in the downward direction from $(i, j)$ to $(n, n)$, and on the left direction from $(i, j)$ to $(n, 1)$ position. If there is no $\Delta$ submatrix created in $M(G)$ for changing the entry from 0 to 1 , then we change the $(i, j)$-th entry from 0 to 1 . Since $M(G)$ is a symmetric matrix, we also change the $(j, i)$-th entry from 0 to 1.

Algorithm 5 returns "True" if $\{u, v\}$ can be inserted into $G$. In that case, we perform the insert operation (see algorithm (4). Consider the strongly chordal graph shown in Figure 6(a). After finding a strong elimination ordering $\left\langle v_{1}, v_{2}, v_{3}, v_{4}, v_{5}, v_{6}, v_{7}\right\rangle$ of $G$, we create the neighborhood 


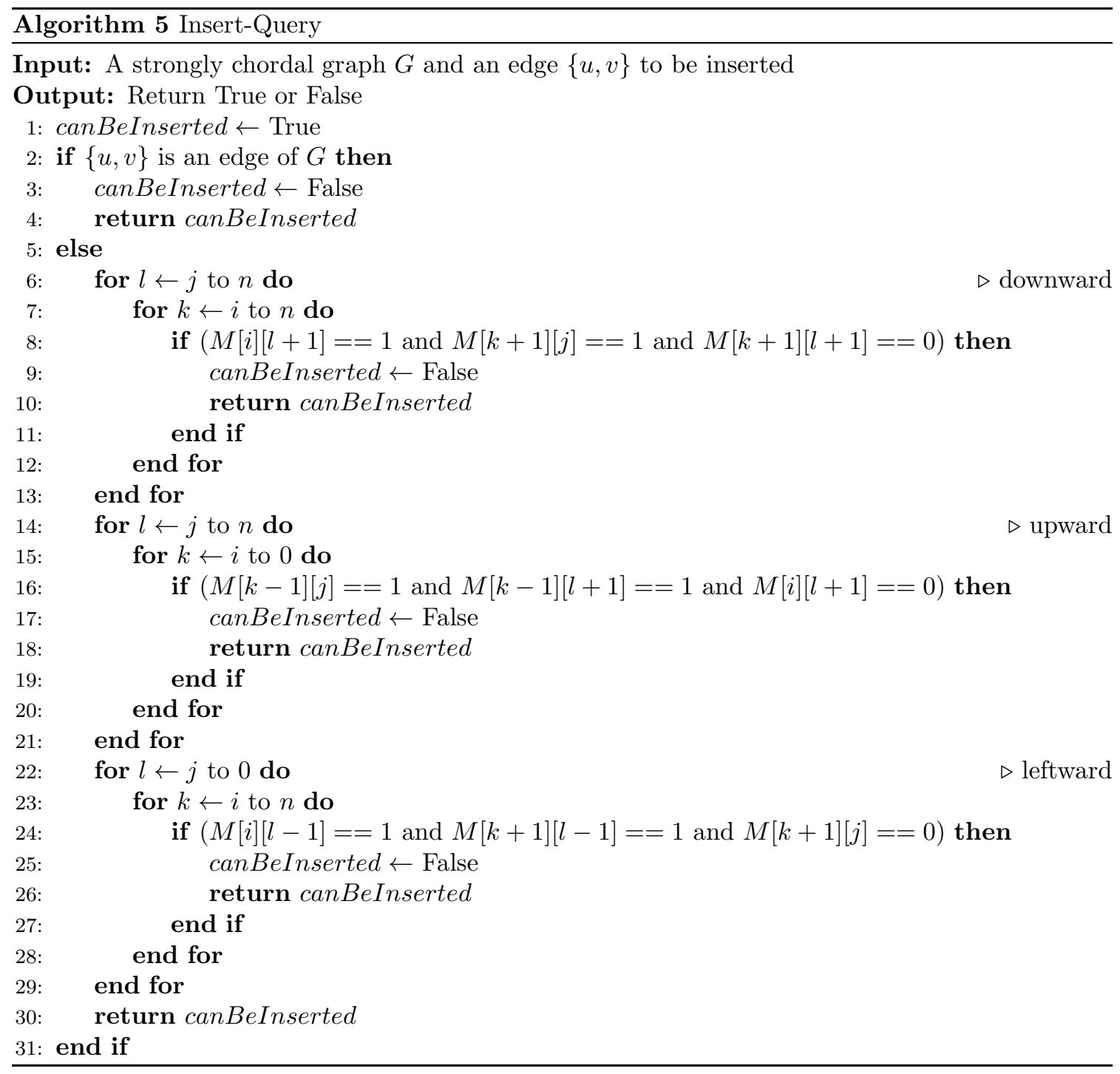

matrix $M(G)$. Now, suppose we want to insert an edge, say $\left\{v_{1}, v_{6}\right\}$, into $G$. However, since the insertion of $\left\{v_{1}, v_{6}\right\}$ creates a $\Delta$ submatrix in $G$, this cannot be done. Next, we might want to insert, say edge $\left\{v_{1}, v_{5}\right\}$, into $G$. This is possible as its insertion does not create any $\Delta$ submatrix in $G$. Since $M(G)$ is a symmetric matrix, we changed 0 to 1 in both symmetric positions (which corresponds to the insertion of $\left\{v_{1}, v_{5}\right\}$ into $G$ ).

\subsection{Complexity of Insertions}

Computing a strong elimination ordering using Farber's algorithm takes $O\left(n^{3}\right)$ time, and it takes $O\left(n^{2}\right)$ time to initialize the neighborhood matrix $M(G)$. Thus the preprocessing time-complexity 
is $O\left(n^{3}\right)$. The upper bound on searching for a $\Delta$ submatrix in $M(G)$ is $O\left(n^{2}\right)$. Thus the timecomplexity of an insert-query is $O\left(n^{2}\right)$.

The insertion of an edge take constant time since we maintain a neighborhood matrix data structure to represent $G$.

\section{Discussion}

In this paper, we have presented semi-dynamic algorithms for deletions and insertions of edges into a strongly chordal graph. The proposed semi-dynamic algorithms are based on two different characterizations of strongly chordal graphs. The deletion algorithm is based on a strong chord characterization, while the insertion algorithm is based on a totally balanced matrix characterization. An interesting and challenging open problem is to come up with an efficient fully dynamic algorithm for this class of graphs.

\section{References}

[1] Blair, J. R. S., And PeYton, B. An introduction to chordal graphs and clique trees. In Graph Theory and Sparse Matrix Computation (New York, NY, 1993), A. George, J. R. Gilbert, and J. W. H. Liu, Eds., Springer New York, pp. 1-29.

[2] Blair, J. R. S., And PeYton, B. An introduction to chordal graphs and clique trees. In Graph Theory and Sparse Matrix Computation (New York, NY, 1993), A. George, J. R. Gilbert, and J. W. H. Liu, Eds., Springer New York, pp. 1-29.

[3] FARBER, M. Applications of 1.p. duality to problems involving independence and domination. PhD thesis, Rutgers University, 1982.

[4] FARBer, M. Characterizations of strongly chordal graphs. Discrete Mathematics 43, 2-3 (1983), $173-189$.

[5] Farber, M. Domination, independent domination, and duality in strongly chordal graphs. Discrete Applied Mathematics 7, 2 (1984), 115-130.

[6] IbARra, L. Fully dynamic algorithms for chordal graphs and split graphs. ACM Trans. Algorithms 4, 4 (2008), 40:1-40:20.

[7] Markenzon, L., Vernet, O., and Araujo, L. H. Two methods for the generation of chordal graphs. Annals OR 157, 1 (2008), 47-60.

[8] Mezzini, M. Fully dynamic algorithm for chordal graphs with $\mathrm{O}(1)$ query-time and $\mathrm{o}\left(\mathrm{n}^{2}\right)$ update-time. Theor. Comput. Sci. 445 (2012), 82-92.

\section{A Strong chords of even cycles of size 6 or greater}

In this appendix, we justify why it is enough to check if an edge $e$ that is a candidate for deletion is a strong chord of a 6 -cycle. 
Definition: Let $C_{k}$ denote a cycle with $k$ edges.

Defintion: An ensemble $\mathcal{E}$ of strong chords of an even cycle with $2 n$ edges, $C_{2 n}$, is a set of $n-2$ strong chords that are pairwise disjoint, except for common end-points.

Two different ensembles of strong chords are shown in Fig. 7 for an 8-cycle, $C_{8}$.

Lemma: A strong chord of a cycle $C_{2 n}$ belongs to an ensemble of $n-2$ strong chords, $\mathcal{E}$.

Proof: Let $v_{1} v_{k}$, for $k \geq 4$ and even, be a strong chord of an even cycle $C_{2 n}=\left\langle v_{1}, v_{2}, \ldots, v_{2 n}\right\rangle$ of length $2 n$, where $n \geq 3$. The proof is by induction on $n$. Clearly as a single strong chord splits a 6 -cycle into two cycles of length 4 , the claim is true for $n=3$. Assume that the claim is true for a cycle of length $2(n-1)$. Since $v_{1} v_{k}$ splits $C_{2 n}$ into two cycles $C_{k}$ and $C_{k^{\prime}}$ of even lengths $k$ and $k^{\prime}=2 n-k+2$, by the inductive hypothesis, there exists a disjoint ensemble of $k / 2-2$ strong chords that partition $C_{k}$ and a disjoint ensemble of $(2 n-k+2) / 2-2$ strong chords that partition $C_{2 n-k+2}$. Since the strong chord $v_{1} v_{k}$ is not counted, the total number of strong chords that partition $C_{2 n}$ is: $k / 2-2+(2 n-k+2) / 2-2+1=n-2$. This proves the assertion.

Theorem: Each of the strong chords in an ensemble $\mathcal{E}$ of strong chords of $C_{2 n}$ is a strong chord of a 6 -cycle.

Proof: Once again the proof is by induction on $n$. Clearly this is true for $C_{6}$, as the ensemble $\mathcal{E}$ has only one strong chord. Assume the claim holds for an even cycle of smaller length. Among the ensemble $\mathcal{E}$ of strong chords of $C_{2 n}$ there is one, say $c_{1}$, that forms a $C_{4}$ with three boundary edges (see Fig. (8). By the inductive hypothesis, in the even cycle formed by the rest of the $2 n-3$ edges and $c_{1}$ each of the strong chords of the residual ensemble $\mathcal{E}-\left\{c_{1}\right\}$ is a strong chord of a 6 -cycle, $C_{6}$. To show that $c_{1}$ is also a strong chord of a 6 -cycle, we observe from Fig. 8 , that $c_{1}$ is a strong chord of the 6-cycle, $\left\langle v_{2 n} v_{1} v_{2} v_{3} v_{4} v_{5}\right\rangle$, or of the 6-cycle $\left\langle v_{2 n} v_{1} v_{2} v_{3} v_{4} v_{5}\right\rangle$ or of the 6-cycle $\left\langle v_{2 n} v_{1} v_{2} v_{3} v_{4} v_{5}\right\rangle$ and $v_{2 n} v_{5}$ or $v_{1} v_{6}$ or $v_{2 n-1} v_{4}$ is a strong chord in $\mathcal{E}-\left\{c_{1}\right\}$. This completes the proof. 


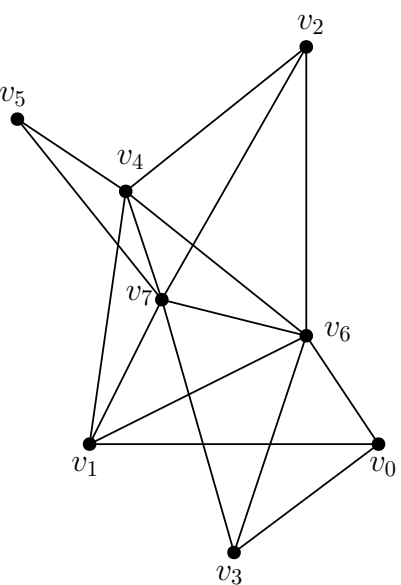

(a) A strongly chordal graph $(G)$

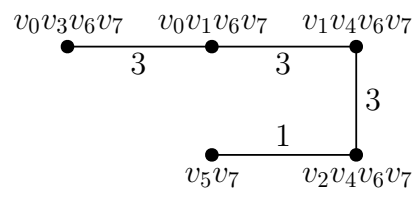

(c) $T-\left\{v_{4}, v_{5}\right\}$

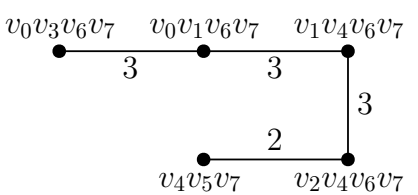

(b) A clique tree $(T)$ of $G$

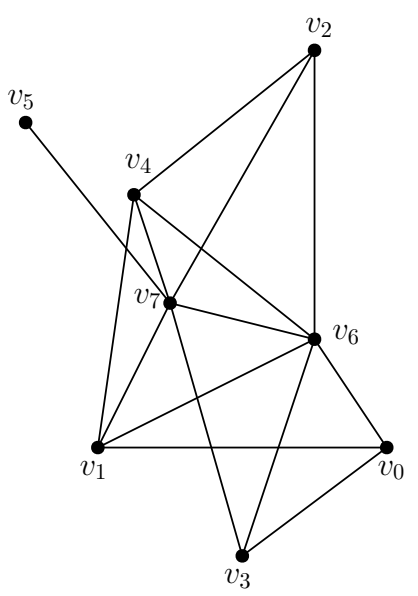

(d) $G-\left\{v_{4}, v_{5}\right\}$

Figure 3: An example

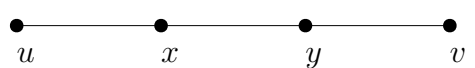

Figure 4: $\mathrm{A}_{4}$ path from $u$ to $v$ 


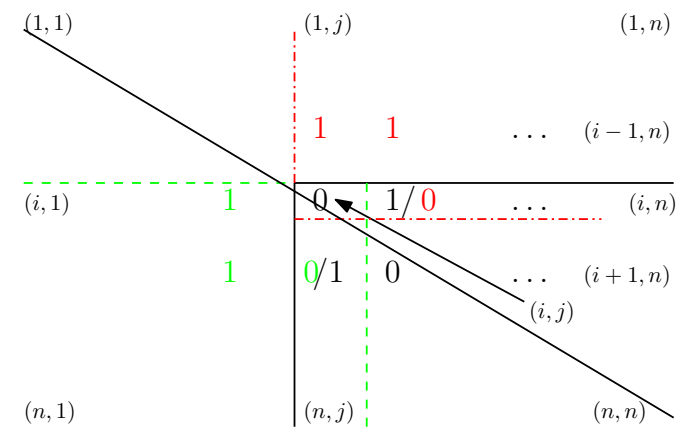

Figure 5: Algorithm to find $\Delta$ submatrix

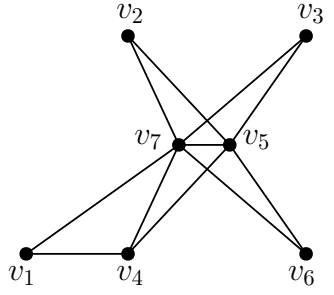

(a) A strongly chordal graph $G$

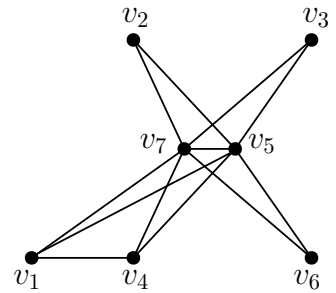

(b) $G+\left\{v_{1}, v_{6}\right\}$

$$
\begin{aligned}
& {\left[\begin{array}{lllllll}
1 & 0 & 0 & 1 & 0 & 0 & 1 \\
0 & 1 & 0 & 0 & 1 & 0 & 1 \\
0 & 0 & 1 & 0 & 1 & 0 & 1 \\
1 & 0 & 0 & 1 & 1 & 0 & 1 \\
0 & 1 & 1 & 1 & 1 & 1 & 1 \\
0 & 0 & 0 & 0 & 1 & 1 & 1 \\
1 & 1 & 1 & 1 & 1 & 1 & 1
\end{array}\right]} \\
& (\mathrm{c}) \\
& \begin{array}{c}
\text { A neighborhood matrix } \\
\text { graph of the strongly chordal }
\end{array}
\end{aligned}
$$

$$
\begin{aligned}
& {\left[\begin{array}{lllllll}
1 & 0 & 0 & 1 & \mathbf{1} & 0 & 1 \\
0 & 1 & 0 & 0 & 1 & 0 & 1 \\
0 & 0 & 1 & 0 & 1 & 0 & 1 \\
1 & 0 & 0 & 1 & 1 & 0 & 1 \\
\mathbf{1} & 1 & 1 & 1 & 1 & 1 & 1 \\
0 & 0 & 0 & 0 & 1 & 1 & 1 \\
1 & 1 & 1 & 1 & 1 & 1 & 1
\end{array}\right]} \\
& (\mathrm{d}) \\
& M(G) \text { neighborhood matrix } \\
& \text { graph shown in Figure (b) }
\end{aligned}
$$

Figure 6: An example of insertion
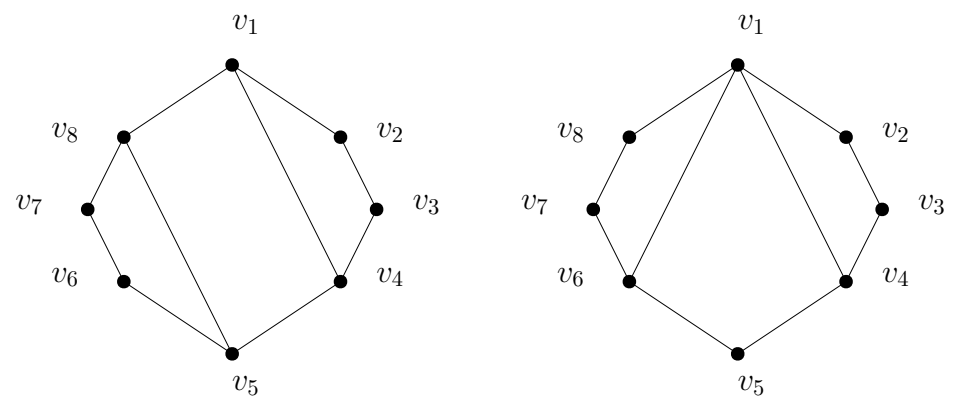

Figure 7: Two different ensembles of strong chords of an 8-cycle 


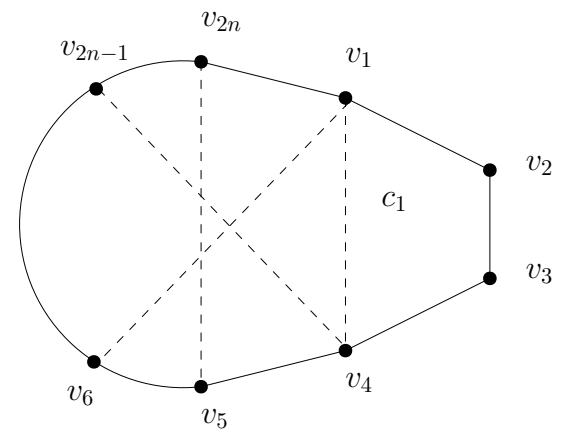

Figure 8: Every strong chord is a strong chord of a 6-cycle 